\title{
New Onset Atrial Fibrillation in Health Profession Student with Newly Diagnosed ADHD: A Case Report on Wellness and Cardio- vascular Consequences of Burnout
}

\author{
Mary Gao ${ }^{1}$, Justin Chin, DO ${ }^{*}\left(\mathrm{D}\right.$, Gayathri Nagendiram ${ }^{1}$, Derek Chen ${ }^{1}$, Parth Rajyaguru, DO and \\ Christine Lomiguen, MD ${ }^{1}$ \\ ${ }^{1}$ Lake Erie College of Osteopathic Medicine, USA \\ ${ }^{2}$ Lifelong Medical Care, USA \\ ${ }^{3}$ Millcreek Community Hospital, USA
}

*Corresponding author: Justin Chin, DO, Lifelong Medical Care, 150 Harbour Way, Richmond, CA 94801, USA

\begin{abstract}
Atrial fibrillation (AF) is an arrhythmia of the heart, typically caused by damage to the myocardium or its electrical pathways, resulting in characteristic "irregularly, irregular" contractions of the atria [1]. It is the most common arrhythmia worldwide, with over 33.5 million people affected [2] Advanced age, male sex, and geographic location have all been linked to increased rates of AF $[1,2]$. According to the American Heart Association, heart disease is the most common risk factor for AF, while hypertension, obesity, smoking, diabetes mellitus, and other cardiac comorbidities have also been shown to play a role [3]. Conversely, lone AF has been used historically to describe AF in patients with structurally and functionally normal hearts [4].
\end{abstract}

\section{Keywords}

Atrial fibrillation, Attention deficit hyperactivity disorder, Methylphenidate, Stimulants, Burnout

Lone $A F$ is typically seen in younger age groups $(<65)$ as many AF risk factors develop with increasing age [4,5]. In general, AF is relatively rare in children and younger patients and is often associated with congenital heart diseases [6]. In the absence of such conditions and when working up the potential for lone AF, it is important for physicians to perform a detailed history and physical exam to determine any exogenous causes [3]. Often overlooked, medications can be a cause of paroxysmal or chronic lone AF, with the most common being corticosteroids, ondansetron, antineoplastic agents, and select cardiovascular drugs such as adenosine, dobutamine, and milrinone [7].
Here we describe a case of methylphenidate-induced lone $\mathrm{AF}$ in a young health professional student that had recently been diagnosed with ADHD, with a review of literature surrounding stimulant use and its relation to wellness and burnout in the health professional education system.

\section{Case Description}

A 23-year-old Caucasian male presented to the emergency department after a 10-minute episode of dizziness, headache with visual aura, shortness of breath, palpitations, and chest pain after exercise, specifically running. Past medical history was significant for recent diagnosis of attention deficit hyperactivity disorder for which he was started on 54 mg Ritalin (extended release methylphenidate) three months prior. He denied any known congenital or structural heart disease, prior cardiac arrhythmias, or other cardiac risk factors. He admitted that he had two cups of coffee prior to running, but denied any recent or past use of alcohol, tobacco, or illicit substances. The patient stated that he never had an episode such as this before, with noncontributory family and surgical history. The patient is a health professions student and cited academic stress as the only constant source for anxiety.

On initial intake and vitals, he was found to have a bounding pulse in the $170 \mathrm{~s}$ and hypotensive blood pressure of $70 / 40$. Physical exam revealed a pleasant,

Citation: Gao M, Chin J, Nagendiram G, Chen D, Rajyaguru P, et al. (2020) New Onset Atrial Fibrillation in Health Profession Student with Newly Diagnosed ADHD: A Case Report on Wellness and Cardiovascular Consequences of Burnout. Int J Clin Cardiol 7:175. doi.org/10.23937/2378-2951/1410175 Accepted: May 04, 2020; Published: May 06, 2020

Copyright: (C) $2020 \mathrm{Gao}$ M, et al. This is an open-access article distributed under the terms of the Creative Commons Attribution License, which permits unrestricted use, distribution, and reproduction in any medium, provided the original author and source are credited. 


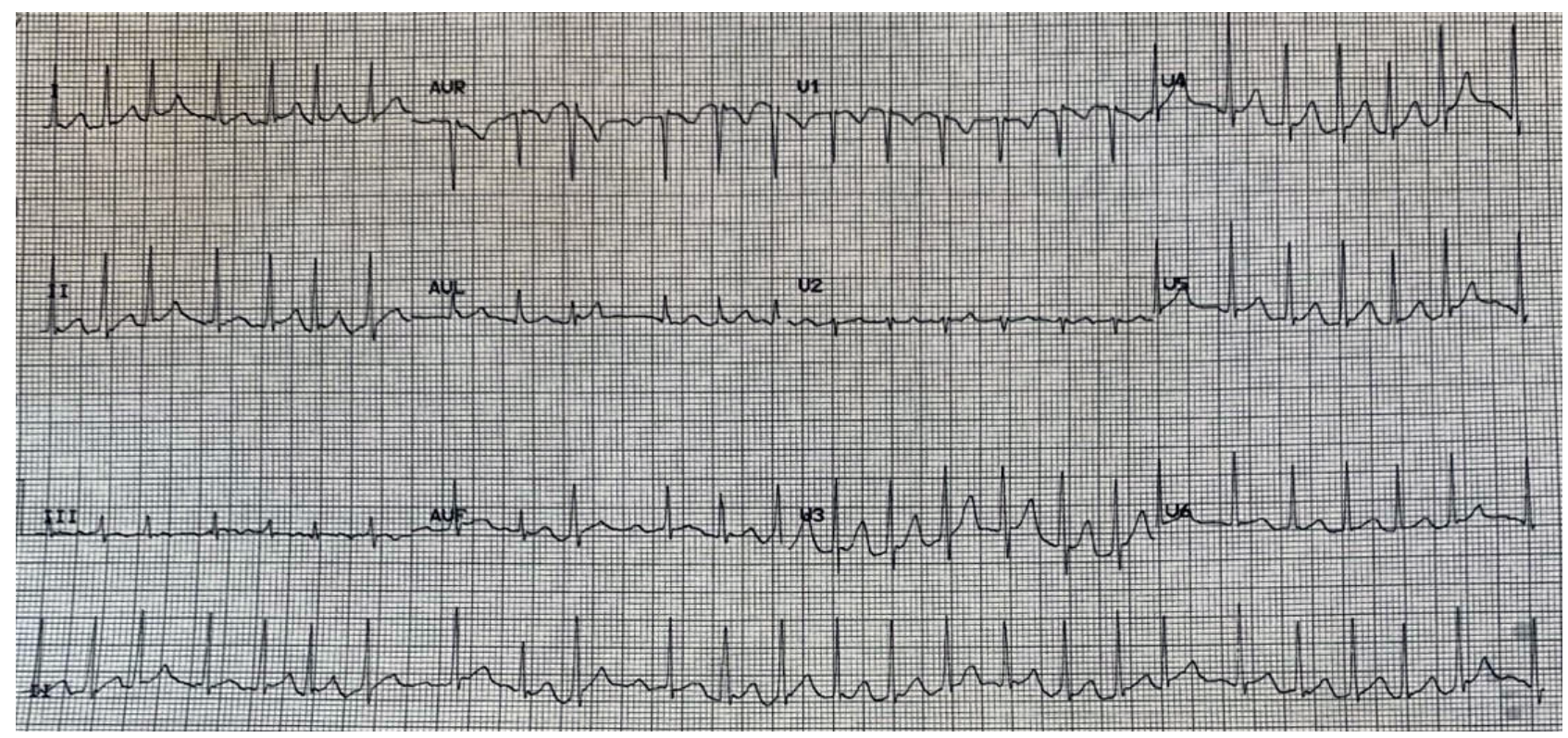

Figure 1: Initial EKG showing irregularly irregular pulse of atrial fibrillation with rapid ventricular response.

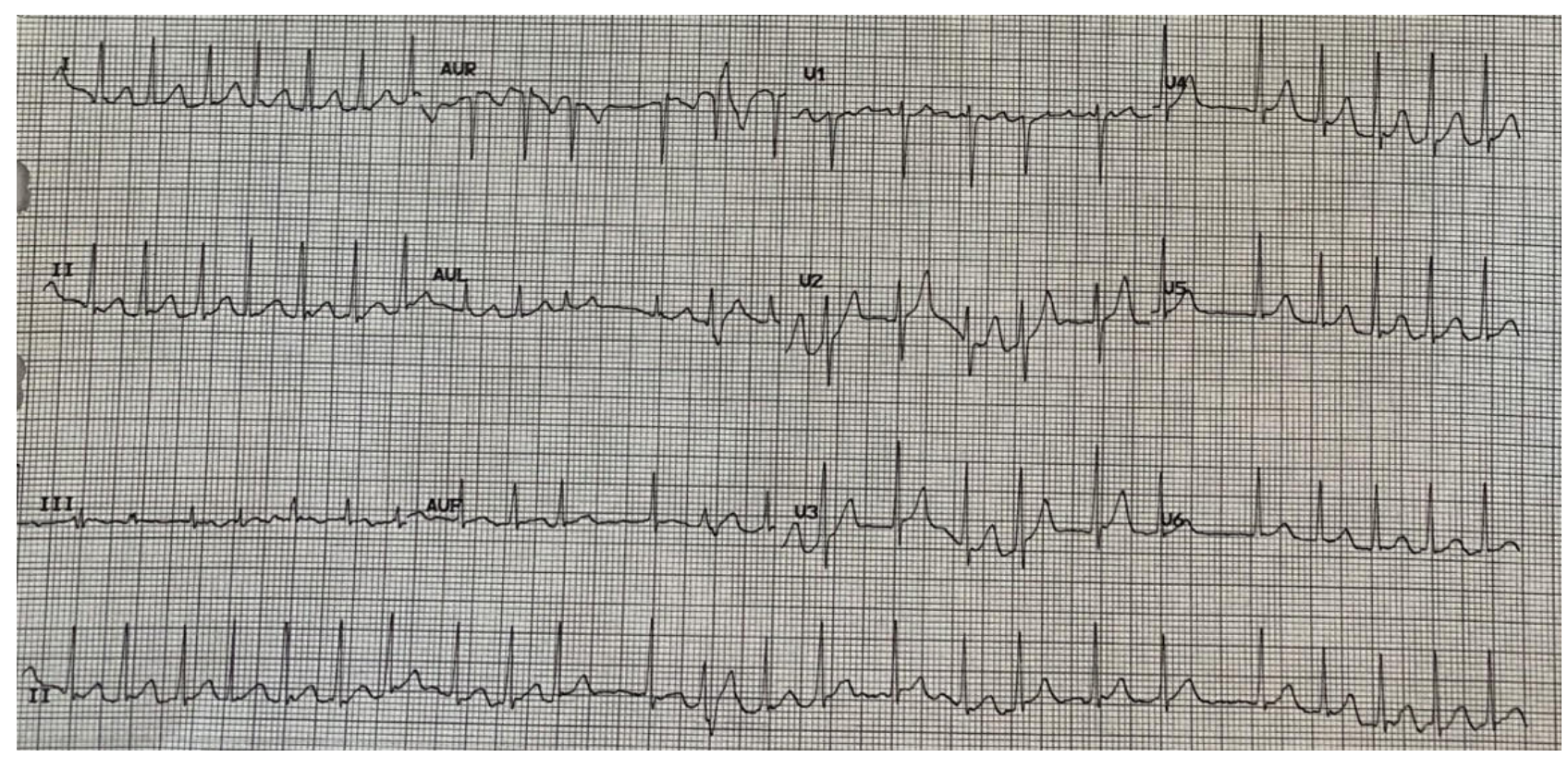

Figure 2: Repeat EKG showing atrial fibrillation with rapid ventricular response despite intravenous fluids and diltiazem.

age-appropriate male with no appreciable gross abnormalities. Cardiovascular exam revealed a normal S1 and S2 on auscultation with no murmurs, gallops, or rubs. Osteopathic structural exam revealed viscerosomatic reflexes visualized at the level of T1-T4.

Initial electrocardiogram revealed atrial fibrillation with rapid ventricular response (Figure 1). Intravenous fluids and two boluses of $10 \mathrm{mg}$ intravenous Cardizem (diltiazem) resulted in a temporary decrease of pulse rate to the $120 \mathrm{~s}$ and blood pressure increase to $108 / 60$. Repeat ECG revealed persistent atrial fibrillation with rapid ventricular response with pulse rate in the $140 \mathrm{~s}$ (Figure 2).

Initial laboratory testing demonstrated a normal complete blood count, serum electrolytes, creatinine, thyroid stimulating hormone, and troponin I. Chest $x$-ray revealed no acute cardiopulmonary findings. Due to his age and relatively healthy status prior to this event, he was placed in observation and given $25 \mathrm{mg}$ Lopressor (metoprolol tartrate). In the next eight hours, repeat troponin levels showed a mild elevation which eventually decreased over a 6 -hour period ( 0.025 to 0.106 to 0.066 ), with spontaneous conversion to normal sinus rhythm in the $70 \mathrm{~s}$ and complete resolution of his initial symptoms. Subsequent transthoracic echocardiogram after sinus conversion revealed normal left ejection fraction of $50-55 \%$, resulting in deferral of a transesophageal echocardiogram.

The patient was discharged on $25 \mathrm{mg}$ Toprol XL (metoprolol succinate) and advised to discontinue his Ritalin until cleared by his primary care physician and outpatient cardiology. With no new cardiac symptoms, 
the patient was restarted on his Ritalin at his 8-week follow-up appointment in addition to continuing with the Toprol XL. At the three-month follow up, he noticed occasional increased heart rate after Ritalin use but has had no recurrent episodes of atrial fibrillation.

\section{Conclusions}

ADHD is a multifactorial neurodevelopmental disorder characterized by difficulty in focusing and enacting actions or emotions without consideration of consequences [8]. Over the past 30 years, the diagnosis and treatment of ADHD and related neuropsychiatric conditions have increased as a reflection of better disease process understanding, changing societal perceptions, and increased awareness [9]. Initial studies have shown a prevalence of $6 \%$ in various populations; however, with revisions to the Diagnostic and Statistical Manual of Mental Disorders and the refinement of stimulants to longer acting formations, current reports have found rates between $10-15 \%$ [8-10]. Although controversial, hereditary and environmental factors have also been studied and implicated in the potential development of ADHD across multiple familial generations [8]. As educators and physicians become more adept in screening and recognition of ADHD, pediatric patients are diagnosed earlier, thus starting stimulants at a younger age and for longer periods of their life [9]. The subsequent effects of stimulants on pediatric cardiophysiology have been inconclusive due to limited studies and small patient pools [4-6].

ADHD is commonly diagnosed in the preadolescent age group, with an average age of 7 years old [8]. In contrast, health profession students are often diagnosed with ADHD later in life due to a combination of adaptive compensation in behavior modulation, fear of stigmatization, and introduction of new life stressors associated with entering the health profession $[11,12]$. ADHD has been shown to be a potential underlying factor to burnout - a syndrome of emotional, physical, and mental exhaustion caused by excessive and prolonged stress that has not been successfully managed $[11,13]$. Recent surveys and anecdotal evidence have detailed rising rates of burnout and suicide in graduate and post-graduate health professions, in which bureaucratic administrative decisions, complicated recordkeeping systems, and quickly evolving requirements create an environment that eliminates a work-life balance and prioritizes efficiency and productivity [12]. Research on the development of response mechanisms in relation to resiliency and wellness increasingly implicate the importance of cultivating healthy habits during the undergraduate health profession years [11-13]. Limited studies have shown that health profession students may utilize stimulants in an effort to improve academic performance; however, no long-term studies exist regarding its effects on cardiovascular health or its use as a coping mechanism $[11,14]$.
Methylphenidate and other stimulant medications can also have similar cardiovascular side effects ranging from use-dependent palpitations to Raynaud's phenomenon and chest pain, but these medications have not been known to precipitate AF [10]. Advances in understanding, screening, and diagnosis of neurodevelopmental and mental health coupled with de-stigmatization of attention deficit hyperactivity disorder (ADHD) has led to increased rates of prescription and utilization of stimulants for treatment $[9,10]$ Greater availability and portrayal in media have also fueled recreation and performance uses for stimulants in undergraduate and graduate education, especially in relation to important examinations [14]. Health profession students, in particular, are not immune from academic stress and the pressure to perform, resulting in the procurement and use of stimulants through legitimate health diagnosis or via third party sources [11]. Minimal literature exists of the health ramifications of stimulant medications in the context of the health professional educational system $[14,15]$.

Research on AF has primarily been focused on the early identification and diagnosis of risk factors as well as the development of novel pharmaceutical agents for treatment $[1,3]$. Due to advances in cardiac imaging and intervention, researchers have strayed away from using lone $A F$, resulting in the inconsistent application and reporting of lone AF in literature [5]. Even in recent case reports and series, lone AF has been a proxy for idiopathic AF and its use has been subject to the discretion of authors, editors, and reviewers, thus making it difficult to compare and draw conclusions $[4,6]$. Coupled with a generally positive prognosis and identical treatment options amongst various AF subtypes, studies have been limited in further elucidating the possible causes of lone AF [5]. In the absence of electrical or structural damage of the heart, lone AF can still be a useful term to describe situations where external stimuli may induce $A F$, such as in the presented case. Methylphenidate and other similar ADHD stimulant medications have not been known to induce AF and further studies should be performed to see if long term or non-prescription base use can precipitate lone AF [10]. Specifically, it is unknown if medication induced lone AF may ultimately increase the risk of developing future cardiac pathology or AF [4-6]. Future studies may look into better quantifying and qualifying ADHD incidence and stimulant use/misuse in health profession students and creating systems to change the culture surrounding burnout and mental health.

Methylphenidate-induced atrial fibrillation is a rare phenomenon, with hallmarks of lone AF due to the lack of damage to cardiac structures or electrical pathways. Rising rates of ADHD due to increased understanding and diagnosis has resulted in greater use of stimulant medications for longer periods of time. Generally safe, stimulant medications have mild cardiovascular side 
effects that are often overlooked relative totreatment benefit and its use as a colloquially known underground study aid in higher education. Health profession students are not excluded from such pressures, however limited data exists. In light of efforts to increase healthcare wellness and career longevity, evaluating the overall environment and its relation to coping behaviors and mechanism may be crucial in preventing adverse events related to burnout and medication misuse.

\section{Conflict of Interest Statement}

The authors have no conflicts to disclose.

\section{Funding Statement}

This research did not receive any specific grant from funding agencies in the public, commercial, or not-forprofit sectors.

\section{References}

1. Zimetbaum P (2017) Atrial Fibrillation. Ann Intern Med 166 : 33-48.

2. Rahman F, Kwan GF, Benjamin EJ (2014) Global epidemiology of atrial fibrillation. Nat Rev Cardiol 11: 639-654.

3. Chen LY, Chung MK, Allen LA, Ezekowitz M, Furie KL, et al. (2018) Atrial fibrillation burden: Moving beyond atrial fibrillation as a binary entity: A scientific statement from the american heart association. Circulation 137: e623-e644.

4. Wyse DG, Van Gelder IC, Ellinor PT, Go AS, Kalman JM, et al. (2014) Lone atrial fibrillation: Does it exist? J Am Coll Cardiol 63: 1715-1723.

5. Scardi S, Mazzone C, Pandullo C, Goldstein D, Poletti A, et al. (1999) Lone atrial fibrillation: Prognostic differences between paroxysmal and chronic forms after 10 years of follow-up. Am Heart J 137: 686-691.
6. Dilaveris PE, Kennedy HL (2017) Silent atrial fibrillation: Epidemiology, diagnosis, and clinical impact. Clin Cardiol 40: 413-418.

7. Kaakeh Y, Overholser BR, Lopshire JC, Tisdale JE (2012) Drug-induced atrial fibrillation. Drugs 72: 1617-1630.

8. Thapar A, Cooper M (2016) Attention deficit hyperactivity disorder. Lancet 387: 1240-1250.

9. Sayal K, Prasad V, Daley D, Ford T, Coghill D (2018) ADHD in children and young people: Prevalence, care pathways, and service provision. Lancet Psychiatry 5:175-186.

10. Hennissen L, Bakker MJ, Banaschewski T, Carucci S, Coghill D, et al. (2017) Cardiovascular effects of stimulant and non-stimulant medication for children and adolescents with ADHD: A systematic review and meta-analysis of trials of Methylphenidate, Amphetamines and Atomoxetine. CNS Drugs 31: 199-215.

11. O'Callaghan P, Sharma D (2014) Severity of symptoms and quality of life in medical students with ADHD. J Atten Disord 18: 654-658.

12. Tuttle JP, Scheurich NE, Ranseen J (2010) Prevalence of ADHD diagnosis and nonmedical prescription stimulant use in medical students. Acad Psychiatry 34: 220-223.

13. Dyrbye LN, Thomas MR, Shanafelt TD (2006) Systematic review of depression, anxiety, and other indicators of psychological distress among U.S. and Canadian medical students. Acad Med 81: 354-373.

14. Arria AM, DuPont RL (2010) Nonmedical prescription stimulant use among college students: Why we need to do something and what we need to do. J Addict Dis 29: 417426.

15. Bossaer JB, Gray JA, Miller SE, Enck G, Gaddipati VC, et al. (2013) The use and misuse of prescription stimulants as "cognitive enhancers" by students at one academic health sciences center. Acad Med 88: 967-971. 